\title{
Avaliação de Características de Crescimento Pós-Desmama de Animais Nelore Puros e Cruzados no Estado do Mato Grosso do Sul ${ }^{1}$
}

\author{
Carolina Amália de Souza Dantas Muniz $2,3,4$, Sandra Aidar de Queiroz ${ }^{3,4}$
}

\begin{abstract}
RESUMO - Com o objetivo de comparar o desempenho de diferentes grupos genéticos para características de crescimento, foram usadas 2888 observações de peso ajustado aos 365 dias (P365) e de ganho médio diário da desmama aos 365 dias (G365) e 1909 observações de peso ajustado aos 550 dias de idade (P550) e de ganho médio diário dos 365 aos 550 dias de idade (G550) de animais F1, oriundos do cruzamento de vacas Nelore com touros Aberdeen Angus, Brangus (pelagens preta e vermelha), Canchim, Gelbvieh, Nelore e Simental, no Estado Mato Grosso do Sul. Os grupos genéticos F1 estudados foram os seguintes: 1/2 Aberdeen Angus + 1/2 Nelore (1AG1NL), 1/2 Brangus (pelagem preta) $+1 / 2$ Nelore (1BA1NL), 1/2 Brangus (pelagem vermelha) $+1 / 2$ Nelore (1BV1NL), 1/2 Canchim $+1 / 2 \mathrm{Nelore}(1 \mathrm{CN} 1 \mathrm{NL})$, 1/2 Gelbvieh + 1/2 Nelore (1GL1NL), 1/2 Simental + 1/2 Nelore (1SM1NL) e Nelore. As análises estatísticas foram feitas pelo método dos quadrados mínimos e o modelo matemático utilizado incluiu os efeitos fixos de grupo contemporâneo (GC), grupo genético do animal (GG), idade do animal e idade da vaca ao parto. Grupo Contemporâneo e Grupo Genético influenciaram significativamente todas as características estudadas. A idade do bezerro foi significativa para G365 e G550. A idade da vaca ao parto apresentou influência linear e quadrática sobre G550. Os animais cruzados foram 25,9 e 34,8 kg mais pesados que os puros Nelore para P365 e P550, respectivamente. Para G365 e G550, os cruzados ganharam, em média, 67 e 21 g por dia, respectivamente, a mais que os puros Nelore. Os animais F1 dos grupos 1AG1NL, 1GL1NL e 1SM1NL foram, aproximadamente, $8 \%$ superiores que demais F1, para as características estudadas, e entre esses, os grupos 1GL1NL e 1SM1NL destacaram-se até os 365 dias de idade. Entre os grupos oriundos de raças sintéticas, 1BA1NL, 1BVNL e 1CN1NL, os cruzados Canchim foram 4 a $7 \%$ superiores para as características estudadas.
\end{abstract}

Palavras-chave: bovinos de corte, cruzamento, ganho médio diário, peso ao ano, peso ao sobreano

\section{Post-Weaning Traits Evaluation of Straightbred and Crossbred Nellore Cattle in Mato Grosso do Sul State}

\begin{abstract}
With the objetive to compare the genetic group performances for growth traits, data on 2888 yearling weight (YW) and average daily gain from weaning to yearling (AGY) and 1909 long yearling weight (LYW) and average daily gain from 365 day weight to 550 day weight (AGL), from progenies of Nellore cows mated to Aberdeen Angus, (red and black) Brangus, Gelbvieh, Nellore and Simmental sires in the State of Mato do Grosso do Sul were analyzed. The F1 genetic groups were: $1 / 2$ Nellore $+1 / 2$ Angus (1AG1NL), 1/2 Brangus + 1/2 Nellore (1BB1NL), 1/2 Red Brangus + 1/2 Nellore (1RB1NL), 1/2 Canchim + 1/2 Nellore (1CN1NL), 1/2 Gelbvieh + 1/2 Nellore (1GL1NL), $1 / 2$ Simental $+1 / 2$ Nellore (1SM1NL) and Nellore. Data were processed by least squares method using a mathematical model that included the fixed effects of contemporary groups (CG), genetic group (GG), age of calf and age of cow at calving. Contemporary Group and Genetic Group showed significant effects on all studied traits. Age of cow at calving was a significant source of variation on AGL, showing linear and quadratic effects. The crossbred animals were $25.9 \mathrm{~kg}$ and $34.8 \mathrm{~kg}$ heavier than the Nellore to YW and LYW, respectively. AGY and AGL were 67 and $21 \mathrm{~g}$ greater for crossbred than for Nellore, respectively. The F1 animals from groups 1AG1NL, 1GL1NL and 1SM1NL were 8\% higher than Nellore for the studied traits. Among crossbreed, 1GL1NL and 1SM1NL tended to be higher for YW. Among the groups originated from sinthetic breeds, 1BA1NL, 1BV1NL and 1CN1NL, the crossbred group Canchim was 4 to $7 \%$ higher for all the traits analysed.
\end{abstract}

Key Words: beef catlle, crossbreding, average daily gain, yearling weight, long yearling weight

\section{Introdução}

O cruzamento entre raças de bovinos de corte vem sendo utilizado como forma rápida de melhorar a eficiência da produção de carne, tanto por propor- cionar a complementaridade entre raças, como detectar manifestação da heterose, sendo esta máxima na primeira geração de cruzamento, se decorrente da dominância. A disponibilidade de grande número de raças de bovinos de corte de diferentes tipos biológi-

\footnotetext{
${ }^{1}$ Parte da dissertação do primeiro autor para obtenção do título de mestre em Zootecnia/Melhoramento Genético Animal na Faculdade de Ciências Agrárias e Veterinárias da UNESP - Jaboticabal - SP.

${ }^{2}$ Aluna de doutorado do curso de Zootecnia - Produção Animal - FCAV - UNESP - Jaboticabal - SP.

${ }^{3}$ Departamento de Zootecnia - FCAV - UNESP - Jaboticabal - SP.

${ }^{4}$ Bolsista CNPq.
} 
cos permite a adequação do animal ao ambiente de produção, pois deve-se considerar que a magnitude da diferença entre puros e cruzados depende, principalmente, do ambiente que é fornecido aos animais, das raças utilizadas e do valor genético dos indivíduos que são acasalados. A distância genética existente entre as raças utilizadas influi diretamente na manifestação da heterose, sendo que a heterose ocasionada pelo acasalamento entre zebu e taurino é cerca de duas vezes a proporcionada por um acasalamento de taurino-taurino ou zebuíno-zebuíno (FRIES, 1996).

Segundo FRISCH (1987), a heterose realizada em determinado ambiente é resultado do potencial de crescimento das raças utilizadas em termos de resistência ao estresse ambiental. Assim, a combinação do alto potencial para crescimento de animais Bos taurus e da alta resistência ao estresse ambiental dos Bos indicus é responsável pela superioridade dos produtos F1. No Brasil, os resultados de cruzamentos empregando-se raças européias (Bos taurus) e raças indianas (Bos indicus) mostram o melhor desempenho dos animais cruzados em relação aos puros, pela maior expressão da heterose, conforme os trabalhos de FRANKE (1980), SHERIDAN (1981) e SYRSTAD (1985). A superioridade dos cruzados sobre os puros Nelore para os pesos aos 365 e 550 dias de idade variou de 9 a $44 \%$ e de 17 a $25 \%$, respectivamente, conforme resultados encontrados na literatura consultada (RIBEIRO et al., 1990; JOSÉ et al., 1991; JOSÉ et al., 1991; e CUBAS et al., 1996) para produtos de fêmeas Nelore com touros de raças européias, criados a pasto.

No Texas, PASCHAL et al. (1994) verificaram que, para características de crescimento pós-desmama, os cruzados F1 de touros zebu com vacas Hereford foram mais pesados que os cruzados F1 de touros Angus com vacas Hereford a um ano de idade, indicando que os cruzados Angus não foram tão bem adaptados às condições de ambiente do Texas Central como os cruzados Zebu, sendo que a diferença foi mais acentuada nos anos com baixa qualidade de forragem. Na África, GREGORY et al. (1985), estudando diferentes grupos genéticos de zebuínos e taurinos, verificaram que os bezerros filhos das vacas $1 / 2$ taurino $+1 / 2$ zebuíno, portanto $3 / 4$ taurino $+1 / 4$ zebuíno, desenvolveram-se melhor até a desmama que bezerros $1 / 2$ zebuíno $+1 / 2$ taurino. Entretanto, no período após a desmama, os bezerros $1 / 2$ taurino + $1 / 2$ zebuíno obtiveram melhor desempenho, em razão da melhor adaptação ao ambiente climático e nutritivo ao qual estavam submetidos. O decréscimo da influência materna sobre o crescimento após a desmama parece coincidir com o aumento da heterose individual (NITTER, 1978).

O objetivo deste trabalho foi comparar o desempenho de diferentes grupos genéticos F1, nas características peso aos 365 dias (P365), ganho médio diário da desmama aos 365 dias (G365), peso aos 550 dias (P550) de idade e ganho médio diário dos 365 aos 550 dias de idade (G550).

\section{Material e Métodos}

Os dados utilizados no presente estudo são referentes a 2888 e 1909 observações do peso ajustado aos 365 dias (P365) e do ganho médio diário da desmama aos 365 dias; do peso ajustado aos 550 dias (P550); e do ganho médio diário dos 365 aos 550 dias de idade (G550) respectivamente, de animais provenientes do cruzamento de vacas Nelore, com touros das raças Aberdeen Angus, Brangus, Canchim, Nelore, Gelbvieh e Simental, ocorridos entre 1991 e 1994, na Fazenda Ivaé, situada no município de Amambai, Mato Grosso do Sul. A fazenda está localizada, aproximadamente, a $23^{\circ} 10^{\prime}$ de latitude sul e $55^{\circ} 15^{\prime}$, de longitude oeste. Caracteriza-se por duas estações climáticas distintas, período das águas, de outubro a março, no qual as chuvas são abundantes, e uma estação seca no período de abril a setembro. Os animais foram criados em pastagens constituídas, na sua grande parte, por brachiárias ( $B$. brizantha, $B$. decumbens, B. ruzizienses), recebendo, apenas, suplementação mineral.

Foram utilizados acasalamentos de vacas Nelore e touros em monta natural (MN) das seguintes raças: Brangus, (pelagens preta e vermelha), Canchim, Nelore e Simental. Em inseminação artificial (IA) foi utilizado sêmen das raças Aberdeen Angus, Canchim, Gelbvieh, Nelore e Simental (Tabela 1).

Os pesos foram ajustados para 365 e 550 dias de idade. O ganho médio diário da desmama aos 365 dias (G365) e dos 365 aos 550 dias (G550) foi obtido pela razão da diferença entre o peso observado aos 365 dias e o peso observado à desmama, pelo número de dias contidos no período compreendido entre as duas pesagens e pela razão da diferença entre o peso observado aos 550 dias e peso observado aos 365 dias, pelo número de dias contidos no período compreendido entre as duas pesagens. Foi criada a variável grupo contemporâneo (GC), para agrupar animais nascidos no mesmo ano e mês, de mesmo sexo, e desmamados na mesma data juliana. Para P365 e 
G365, os nascimentos ocorreram de 1991 a 1994, nos meses de janeiro, fevereiro, julho, setembro, outubro, novembro e dezembro, sendo verificados 39 grupos de desmama na mesma data juliana. Para P550 e G550, os nascimentos ocorreram de 1991 a 1993, nos mesmos meses já citados, sendo verificados 31 grupos de desmama. Grupos contemporâneos compostos por menos que cinco animais foram descartados da análise.

Para comparar o desempenho dos grupos genéti$\cos$ nas diferentes idades, as observações foram analisadas pelo Método dos Quadrados Mínimos, utilizando-se o procedimento GLM do SAS (1992), pelo seguinte modelo fixo:

$$
\begin{gathered}
\mathrm{Y}_{\mathrm{ijklm}}=\mu+\mathrm{GC}_{\mathrm{i}}+\mathrm{GG}_{\mathrm{j}}+\mathrm{b}_{1}\left(\mathrm{I}_{\mathrm{ijk}}\right)+\mathrm{b}_{2}\left(\mathrm{x}_{\mathrm{ijkl}}\right)+ \\
\mathrm{b}_{3}\left(\mathrm{x}_{\mathrm{ijkl}}\right)^{2}+\mathrm{e}_{\mathrm{ijklm}}
\end{gathered}
$$

em que

$\mathrm{Y}_{\mathrm{ijklm}}=$ observação (desempenho) do m-ésimo animal;

$\mu \quad=$ média geral;

$\mathrm{GC}_{\mathrm{i}}=$ efeito fixo do i-ésimo grupo contemporâneo (i $\leq 75)$;

$\mathrm{GG}_{\mathrm{j}} \quad=$ efeito fixo do $\mathrm{j}$-ésimo grupo genético $(\mathrm{j}=1, \ldots 7)$;

$\mathrm{b}_{1} \quad=$ coeficiente de regressão linear;

$\mathrm{I}_{\mathrm{ijk}} \quad=$ efeito fixo da k-ésima idade do animal na data da respectiva pesagem, em dias;

$b_{2}$ e $b_{3}=$ coeficientes de regressão linear e quadrático, respectivamente;

$\mathrm{x}_{\mathrm{ijkl}}=$ efeito fixo da l-ésima idade da vaca ao parto em anos $(1=3, \ldots 11)$;

$\mathrm{e}_{\mathrm{ijklm}}=$ erro aleatório associado a cada observação ijklm, suposto normalmente distribuído com média zero e variância $\sigma^{2}$.

Foram também realizadas análises estatísticas separadas de acordo com o tipo de acasalamento (IA ou $\mathrm{MN}$ ), utizando-se o mesmo modelo descrito anteriormente. As médias dos diferentes grupos genéticos foram comparadas utilizando-se contrastes ortogonais (SAS, 1992). Assim, os contrastes entre as médias estimadas para os grupos genéticos nas diferentes análises não foram os mesmos, pois existiam touros que foram utilizados apenas em inseminação artificial e outros apenas em monta natural (Tabela 1).

\section{Resultados e Discussão}

O resumo das análises de variância das características estudadas é apresentado na Tabela 2. Verifica-se que GC e GG influenciaram significativamente $(\mathrm{P}<0,001)$ todas as características estudadas. O efeito da idade do animal não foi significativo para P550, o que indica que a padronização foi suficiente para ajustar os pesos para esta idade. Entretanto, para P365 o efeito da idade do produto foi significativo, possivelmente devido à maior variação na idade de pesagem dos animais a um ano de idade. A idade do animal também apresentou efeito significativo sobre G550. Estes resultados indicam a necessidade de fatores de correção específicos, tanto para os pesos às idades-padrão, como para o ganho médio diário nos períodos estudados. Entretanto, considera-se que a inclusão da idade do produto no modelo tenha eliminado as variações devido a este efeito. A idade da vaca ao parto não apresentou efeitos significativos para P365, G365 e P550. No período após a desmama, o efeito da idade da vaca ao parto não é tão expressivo como à desmama. Tornando-se independente da mãe, o desempenho do bezerro nesta fase, possivelmente, está mais ligado à expressão de seu potencial genético para crescimento. Porém, obser-

Tabela 1- Número de touros usados no período de 1991 a 1994, de acordo com a raça e o tipo de acasalamento

\begin{tabular}{|c|c|c|c|}
\hline \multirow{3}{*}{$\begin{array}{l}\text { Raça do touro } \\
\text { Breed of sire }\end{array}$} & \multicolumn{2}{|c|}{ Tipo de acasalamento } & \multirow{3}{*}{ Total } \\
\hline & Inseminacão artificial & Monta natural & \\
\hline & Artificial insemination & Natural service & \\
\hline Nelore & 2 & 32 & 34 \\
\hline Aberdeen Angus & 5 & - & 5 \\
\hline Brangus (pelagem preta) & - & 10 & 10 \\
\hline \multicolumn{4}{|l|}{ Black Brangus } \\
\hline Brangus (pelagem vermelha) & - & 6 & 6 \\
\hline \multicolumn{4}{|l|}{ Red Brangus } \\
\hline Canchim & 4 & 8 & 12 \\
\hline Gelbvieh & 5 & - & 5 \\
\hline Simental & 5 & 6 & 11 \\
\hline
\end{tabular}

Table 1 - Number of sires used from 1991 to 1994, according to the breed and type of service 
Tabela 2- Quadrado médio para peso ajustado aos 365 dias (P365), ganho médio diário da desmama aos 365 dias (G365), peso ajustado aos 550 dias de idade (P550) e ganho médio diário da desmama aos 550 dias de idade (G550) de bovinos Nelore e cruzados

Table 2 - Mean squares for weight adjusted to 365 days (W365), average daily gain from weaning to 365 days (G365), weight adjusted to 550 days of age (W550) and average daily gain from weaning to 550 days of age (G550) of Nellore and crossbred cattle

\begin{tabular}{|c|c|c|c|c|}
\hline \multirow[b]{2}{*}{ Fonte de variação } & \multicolumn{4}{|c|}{$\begin{array}{l}\text { Quadrado médio }(\mathrm{gl}) \\
\text { Mean square }(d f)\end{array}$} \\
\hline & P365 & G365 & P550 & G550 \\
\hline Source of variation & W365 & G365 & W550 & G550 \\
\hline Grupo contemporâneo & $12830,00 * * *(74)$ & $0,9394 * * *(74)$ & $19375,91 * * *(59)$ & $0,3237 * * *(59)$ \\
\hline Contemporary group & & & & \\
\hline Grupo genético & $49409,99 * * *(6)$ & $0,2602 * * *(6)$ & $55126,20 * * *(6)$ & $0,1263 * * *(6)$ \\
\hline $\begin{array}{l}\text { Genetic Group } \\
\text { Idade do animal (L) } \\
\text { Age of the animal }(L)\end{array}$ & $34088,73 * * *(1)$ & $0,0499(1)$ & $165,52(1)$ & $0,1606 * * *(1)$ \\
\hline $\begin{array}{l}\text { Idade da vaca ao parto (L) } \\
\text { Age of the cow at calving (L) }\end{array}$ & $2066,64(1)$ & $0,0218(1)$ & $18,88(1)$ & $0,0894(1)$ \\
\hline $\begin{array}{l}\text { Idade da vaca ao parto }(\mathrm{Q}) \\
\text { Age of the cow at calving }(Q)\end{array}$ & $1991,19(1)$ & $0,0115(1)$ & $110,84(1)$ & $0,0991 * *(1)$ \\
\hline Resíduo & $508,60(2804)$ & $0,0153(2804)$ & $825,21(1840)$ & $0,0123(1840)$ \\
\hline
\end{tabular}

Error

$\mathrm{R}^{2}(\%)$

51

64

59

53

${ }^{* *}=\mathrm{P}<0,01 ;={ }^{* \star *} \mathrm{P}<0,001$.

$\mathrm{L}=$ linear, $\mathrm{Q}=$ quadrática.

$\mathrm{L}=$ linear, $\mathrm{Q}=$ quadratic.

vou-se efeito quadrático da idade da vaca ao parto sobre G550. A Figura 1 mostra a tendência observada da variável G550, em função da idade da vaca. Vacas entre seis e oito anos de idade produziram os bezerros com menor ganho médio diário dos 365 aos 550 dias de idade, enquanto as vacas mais jovens, de três a quatro anos de idade ou mais velhas, de 10 a 11 anos de idade, produziram os bezerros com maior ganho médio diário. Os resultados observados podem estar indicando a ocorrência de crescimento compensatório dos bezerros nesse período. As vacas mais jovens ou mais velhas do rebanho, que desmamaram bezerros cruzados com maior capacidade de ingestão de leite, possivelmente, não tiveram condições de atender suas exigências nutricionais durante o período de amamentação e, assim, desmamaram bezerros mais leves que as vacas em melhores condições de produção de leite (entre 7 e 9 anos de idade). CAMPOS e FRIES (1989), analisando o efeito da idade da vaca ao parto no crescimento dos bezerros no período após a desmama, verificaram efeitos lineares e quadráticos da idade da vaca ao parto sobre os pesos aos 365, 410 e 550 dias de idade, em animais das raças Angus, Devon e Hereford. Bezerros filhos de vacas jovens ou de vacas mais velhas, que provavelmente produziram menos leite, ganharam mais peso no período após a desmama. Entretanto, no presente trabalho, deve-se considerar

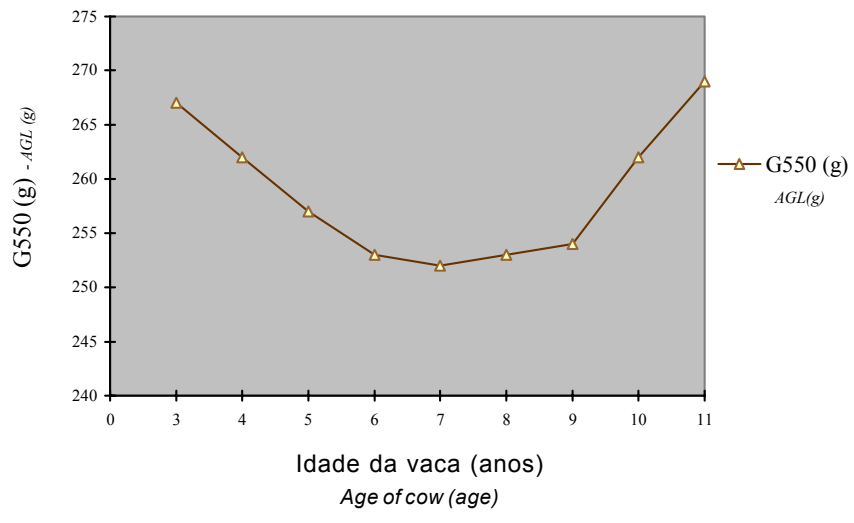

Figura 1 - Regressão do ganho médio diário de peso (g) de bezerros Nelore e cruzados dos 365 aos 550 dias de idade (G550), em relação á idade da vaca ao parto.

Figure 1 - Regression of crossbred and Nellore calves average daily gain $(\mathrm{g})$ from 365 to 550 days of age (AGL), on the age of cows at calving.

também possível confundimento entre a idade da vaca e o grupo genético do animal. Possivelmente, nos acasalamentos, vacas de determinadas idades foram acasaladas, preferencialmente, com touros de determinada raça; por exemplo, vacas de primeira cria inseminadas, principalmente, com touros Aberdeen Angus.

As médias estimadas para P365, G365, P550 e G550, de acordo com o grupo genético do animal, são 
Rev. bras. zootec.

Tabela 3 - Médias estimadas e número de observações ${ }^{1}$ dos pesos ajustados aos 365 (P365) e 550 dias de idade (P550) e dos ganhos médios diários da desmama aos 365 dias (G365) e dos 365 aos 550 dias de idade (G550), conforme o grupo genético do animal

Table 3 - Least squares means, and number of observations ${ }^{1}$ of weight adjusted to 365 days (W365), average daily gain from weaning to yearling(W365), weight adjusted to 550 days (W550) and average daily gain from 365 to 550 days weight (G550), according to the genetic group of the animal

\begin{tabular}{|c|c|c|c|c|}
\hline \multirow[t]{3}{*}{$\begin{array}{l}\text { Grupo genético }^{2} \\
\text { Genetic group }\end{array}$} & \multicolumn{4}{|c|}{$\begin{array}{l}\text { Médias estimadas } \pm \text { Erros-padrão } \\
\text { Least square means } \pm \text { Standard error }\end{array}$} \\
\hline & P365 & G365 & P550 & G550 \\
\hline & W365 & G365 & W550 & $G 550$ \\
\hline Nelore & $\begin{array}{c}200,7 \pm 1,19 \\
(596)\end{array}$ & $\begin{array}{c}211 \pm 6 \\
(596)\end{array}$ & $\begin{array}{c}275,0 \pm 2,21 \\
(317)\end{array}$ & $\begin{array}{c}441 \pm 9 \\
(317)\end{array}$ \\
\hline 1AG1NL & $\begin{array}{l}230,0 \pm 1,53 \\
(347)\end{array}$ & $\begin{array}{c}278 \pm 8 \\
(347)\end{array}$ & $\begin{array}{l}318,1 \pm 2,52 \\
(229)\end{array}$ & $\begin{array}{l}469 \pm 10 \\
(229)\end{array}$ \\
\hline 1BA1NL & $\begin{array}{c}214,7 \pm 1,24 \\
(496)\end{array}$ & $\begin{array}{c}273 \pm 7 \\
(496)\end{array}$ & $\begin{array}{c}288,5 \pm 2,00 \\
\text { (349) }\end{array}$ & $\begin{array}{c}416 \pm 8 \\
(349)\end{array}$ \\
\hline 1BV1NL & $\begin{array}{c}218,0 \pm 1,78 \\
(192)\end{array}$ & $\begin{array}{c}252 \pm 10 \\
(192)\end{array}$ & $\begin{array}{c}297,3 \pm 2,66 \\
(150)\end{array}$ & $\begin{array}{c}448 \pm 10 \\
(150)\end{array}$ \\
\hline $1 \mathrm{CN} 1 \mathrm{NL}$ & $\begin{array}{c}226,0 \pm 1,12 \\
(541)\end{array}$ & $\begin{array}{c}276 \pm 6 \\
(541)\end{array}$ & $\begin{array}{c}309,0 \pm 1,87 \\
(350)\end{array}$ & $\begin{array}{c}464 \pm 7 \\
(350)\end{array}$ \\
\hline 1GL1NL & $\begin{array}{c}238,5 \pm 1,48 \\
(321)\end{array}$ & $\begin{array}{c}289 \pm 8 \\
(321)\end{array}$ & $\begin{array}{c}327,4 \pm 2,32 \\
(230)\end{array}$ & $\begin{array}{c}495 \pm 9 \\
(230)\end{array}$ \\
\hline 1SM1NL & $\begin{array}{c}235,1 \pm 1,32 \\
(395)\end{array}$ & $\begin{array}{c}304 \pm 7 \\
(395)\end{array}$ & $\begin{array}{c}317,7 \pm 2,02 \\
(284)\end{array}$ & $\begin{array}{c}472 \pm 8 \\
(284)\end{array}$ \\
\hline Total & 2888 & 2888 & 1909 & 1909 \\
\hline $\begin{array}{l}\text { Média geral } \\
\text { Overall mean }\end{array}$ & 216,0 & 261,0 & 295,3 & 430,0 \\
\hline
\end{tabular}

apresentadas na Tabela 3 e alguns contrastes de interesse entre as médias são apresentados na Tabela 4. As médias gerais estimadas para P365, G365, P550 e G550 foram iguais a 216,0 kg; $261 \mathrm{~g} ; 295,3 \mathrm{~kg}$; e $430 \mathrm{~g}$, respectivamente. A superioridade dos cruzados sobre os puros Nelore variou de 6 a $19 \%$ e 5 a 19\% para P365 e P550, respectivamente. Para G365 e G550, essa diferença foi de 16 a $44 \%$ e 1 a $12 \%$, respectivamente. Verificou-se maior variação para o ganho médio diário da desmama aos 365 dias de idade (G365). A maior variação do desempenho dos animais neste período deve-se, provavelmente, ao estresse da desmama e, ainda, ao tipo de forragem fornecido ao animal neste período. Os bezerros que são desmamados e encontram pasto inferior em quantidade e qualidade sofrem maior estresse em relação aos que são desmamados em melhores condições de pastagem. O ganho médio diário variou de -538 a 798 g, o que reflete a grande variação que ocorreu no desempenho dos animais para esta característica. Neste período houve a maior diferença entre puros e cruzados (Contraste $\mathrm{C} 1$, Tabela 4). $\mathrm{O}$ ganho médio diário dos 365 aos 550 dias (G550) apresentou a menor diferença entre cruzados e puros (Contraste C1, Tabela 4). Entretanto, de forma geral, maiores valores foram encontrados na comparação dos demais grupos, evidenciando o diferente potencial de crescimento entre os grupos genéticos estudados.

O contraste entre as médias de animais das raças européias e das raças sintéticas evidenciou que os animais dos grupos 1AG1NL, 1GL1NL e 1SM1NL foram mais pesados e ganharam mais peso $(\mathrm{P}<0,001)$ que os grupos 1BA1NL, 1BV1NL e 1CN1NL (Contraste $\mathrm{C} 2$, Tabela 4), nas idades analisadas. Entretanto, deve-se considerar, além da maior heterozigose proveniente do cruzamento entre vacas Nelore e touros Aberdeen Angus, Gelbvieh e Simental, o potencial genético dos touros Aberdeen Angus, Gelbvieh e Simental, possivelmente melhor, uma vez que para essas raças foram utilizados, na sua maior parte, touros provados, em inseminação artificial, enquanto, para o grupo de raças sintéticas, apenas a raça Canchim contou com touros de inseminação artificial.

Os grupos de raças continentais, 1GL1NL e 1SM1NL, foram semelhantes em desempenho para P365 e G365. Para P550 os animais 1GL1NL obtive- 
Tabela 4- Contrastes entre as médias estimadas para os pesos ajustados aos 365 (P365) e 550 dias de idade (P550) e para os ganhos médios diários da desmama aos 365 dias (G365) e dos 365 aos 550 dias de idade (G550) de bovinos Nelore e cruzados

Table 4 - Contrasts among groups of means for weight adjusted to 365 days (W365) and 550 days of age (W550) and for the average daily gain from weaning to 365 days (G365) and from 365 to 550 days of age (G550) of Nellore and crossbred cattle

\begin{tabular}{|c|c|c|c|c|}
\hline Contraste & P365 & G365 & P550 & G550 \\
\hline Contrast & W365 & G365 & W550 & G550 \\
\hline C1. Cruzados ${ }^{1}$ vs. Nelore & $26 * * *$ & $67 * * *$ & $35 * * *$ & 21 \\
\hline $\begin{array}{l}\text { C1. Crossbreed vs Nellore } \\
\text { C2. }(1 \mathrm{GL} 1 \mathrm{NL}+1 \mathrm{SM} 1 \mathrm{NL}+1 \mathrm{AG} 1 \mathrm{NL}) \mathrm{vs} \text {. demais } \mathrm{F} 1^{2} \\
\text { C2. }(1 G L 1 N L+1 S M 1 N L+1 A G 1 N L) \text { vs. others } F 1^{2}\end{array}$ & $15 * * *$ & $23 * * *$ & $23 * * *$ & $36 * * *$ \\
\hline C3. 1GL1NL vs. 1SM1NL & 3 & -15 & $10 * * *$ & 23 \\
\hline C4. (1GL1NL+1SM1NL) vs 1AG1NL & $6 * * *$ & 18 & 4 & 14 \\
\hline C5. 1CN1NL vs. (1BA1NL+1BV1NL) & $10 * * *$ & 14 & $16^{* * *}$ & $32 * * *$ \\
\hline C6.1BV1NLvs.1BA1NL & 4 & -22 & $9 * * *$ & $29 * * *$ \\
\hline
\end{tabular}

${ }^{* *}=p<0,01 ;{ }^{* * *}=p<0,001$.

$11 / 2$ Aberdeen Angus + 1/2 Nelore (1AG1NL), 1/2 Brangus (pelagem preta) + 1/2 Nelore (1BA1NL), 1/2 Brangus (pelagem vermelha) $1 / 2$ Nelore (1BV1NL), 1/2 Canchim + 1/2 Nelore (1CN1NL), 1/2 Gelbvieh + 1/2 Nelore (1GL1NL), 1/2 Simental + 1/2 Nelore (1SM1NL).

2 = 1BA1NL, $1 \mathrm{BV} 1 \mathrm{NL}$ e $1 \mathrm{CN} 1 \mathrm{NL}$.

1 1/2 Aberdeen Angus + 1/2 Nellore (1AG1NL), 1/2 Black Brangus + 1/2 Nellore (1BA1NL), 1/2 Red Brangus + 1/2 Nellore (1BV1NL), 1/2 Canchim + 1/2 Nellore (1CN1NL), 1/2 Gelbvieh + 1/2 Nellore (1GL1NL), 1/2 Simmental + 1/2 Nellore (1SM1NL).

ram maior média, porém a diferença a favor dos animais 1GL1NL não foi significativa para o ganho médio diário dos 365 aos 550 dias de idade (G550). O maior estresse da desmama aos 365 dias de idade poderia impor restrição ao crescimento dos animais, principalmente das raças de grande potencial para crescimento, diminuindo assim a diferença entre esses dois grupos. Além disso, a diferença no desempenho entre os cruzados Gelbvieh e Simental foi menor quando comparados com outros grupos genéticos (Tabela 3), evidenciando a similaridade no desempenho destes dois grupos.

Ao se comparar o desempenho do grupo 1AG1NL com o dos grupos 1GL1NL e 1SM1NL (Contraste C4, Tabela 4), verifica-se que o grupo $1 \mathrm{AG} 1 \mathrm{NL}$ foi mais leve que os grupos de raças continentais Gelbvieh e Simental aos 365 dias de idade (P365). Entretanto, a diferença entre os grupos cruzados $1 \mathrm{AG} 1 \mathrm{NL}$, 1GL1NL e 1SM1NL não foi significativa para G365, P550 e G550. Provavelmente, o maior peso aos 365 dias dos grupos provenientes das raças continentais seja atribuído às vantagens que os mesmos trazem por serem desmamados com maior peso. Ao sobreano, possivelmente, os cruzados de raças continentais necessitam de maior quantidade de nutrientes, para poderem expressar, totalmente, seu potencial genético para crescimento. Assim, na ausência de condições mais favoráveis de ambiente não diferem significativamente dos cruzados Aberdeen Angus.

Verifica-se (Tabela 3) que, entre os sintéticos, os cruzados Canchim (1CN1NL) mostraram a maior diferença em relação à raça Nelore. A diferença entre os grupos de raças sintéticas foi significativa (Contraste C5, Tabela 4) nas idades analisadas, sendo que o grupo $1 \mathrm{CN} 1 \mathrm{NL}$ obteve os maiores pesos e os maiores ganhos médios diários, principalmente aos 550 dias de idade, em que essa diferença foi de $16 \mathrm{~kg}$ para P550. Esse resultado pode estar associado ao potencial genético dos touros Canchim utilizados e, também, ao fato de a raça Canchim ser originada de uma raça continental, o que proporcionaria maiores peso e ganho de peso. Os sintéticos derivados de raças britânicas foram mais leves e ganharam menos peso que os demais grupos cruzados, em todas as idades (Tabela 3). Entre 1BA1NL e 1BV1NL (contraste C6, Tabela 4), o grupo 1BV1NL destacou-se ao sobreano, obtendo as melhores médias. Este resultado pode estar associado ao potencial genético dos touros Brangus de pelagem vermelha utilizados no acasalamento com as vacas Nelore, possivelmente, maior que o potencial genético dos touros Brangus de pelagem preta.

Pelas análises de variância, ao considerar apenas produtos dos touros de IA, verificou-se que o efeito de grupo genético só foi significativo para P365 $(\mathrm{P}<0,001)$. O efeito de grupo genético decresceu de importância, possivelmente, porque o desempenho dos grupos genéticos 1SM1NL, 1GL1NL e 1AG1NL foram semelhantes. Em virtude de os grupos Nelore e 1CN1NL contarem com poucas observações, os outros efeitos considerados no modelo, principalmente o efeito de grupo contemporâneo (GC), foram mais importantes, sendo significativo $(\mathrm{P}<0,001)$ para todas 
Tabela 5 - Contrastes entre as médias estimadas para os pesos ajustados aos 365 dias (P365) de bovinos Nelore e cruzados, para a progênie de touros usados em inseminação artificial

Table 5 - $\quad$ Contrasts among groups of means for weight adjusted to 365 days (W365) of Nellore and crossbred cattle, for progenie of sires used in the artificial insemination

\begin{tabular}{|c|c|}
\hline Contraste & P365 (kg) \\
\hline Contrast & W365 \\
\hline C1. Cruzados ${ }^{1}$ vs. Nelore & $30 * * *$ \\
\hline C1. Crossbreed vs Nellore & \\
\hline C2.1GL1NL vs. 1SM1NL & 3 \\
\hline C3. (1GL1NL+1SM1NL) vs 1AG1NL & $7 * *$ \\
\hline C4. 1CN1NL vs. 1AG1NL & 6 \\
\hline \multicolumn{2}{|c|}{$\begin{array}{l}1 / 2 \text { Aberdeen Angus }+1 / 2 \text { Nelore (1AG1NL), } 1 / 2 \text { Canchim }+1 / 2 \\
\text { Nelore (1CN1NL), } 1 / 2 \text { Gelbvieh }+1 / 2 \text { Nelore (1GL1NL), } 1 / 2 \text { Simental } \\
+1 / 2 \text { Nelore (1SM1NL). } \\
11 / 2 \text { Aberdeen Angus }+1 / 2 \text { Nellore (1AG1NL), } 1 / 2 \text { Canchim }+1 / 2 \text { Nellore } \\
\text { (1CN1NL), } 1 / 2 \text { Gelbvieh }+1 / 2 \text { Nellore (1GL1NL), 1/2 Simmental + 1/2 Nellore } \\
\text { (1SM1NL) }\end{array}$} \\
\hline
\end{tabular}

as características estudadas. Para P365, o contraste que compara o desempenho dos cruzados vs. Nelore (Contraste C1, Tabela 5) foi estatisticamente significativo a favor dos cruzados. O desempenho dos dois grupos de raças continentais não foi significativo (Contraste C2, Tabela 5), o que pode estar indicando, como no resultado anterior, a semelhança no desempenho desses grupos genéticos. Os cruzados de raças continentais foram mais pesados a um ano de idade que os cruzados de raças britânicas (Contraste C3, Tabela 5). Este contraste (Contraste C3, Tabela 5) é, praticamente, o mesmo que o obtido do arquivo que considerou IA e MN juntos, pois as raças Aberdeen Angus e Gelbvieh contam apenas com touros de inseminação artificial e o número de observações é

Tabela 6 - Contrastes entre as médias estimadas para os pesos ajustados aos 365 (P365) e 550 dias de idade (P550) e para os ganhos médios diários da desmama aos 365 dias (G365) e dos 365 aos 550 dias de idade (G550) de bovinos Nelore e cruzados, para a progênie de touros utilizados em monta natural

Table 6 - Contrasts among groups of means for weight adjusted to 365 days (W365) and 550 days of age (W550) average daily gain from weaning to 365 days (G365) and from 365 to 550 days of age (G550) of Nellore and crossbred cattle, for progenie of sires used in the natural

\begin{tabular}{|c|c|c|c|c|}
\hline Contraste & P365 & G365 & P550 & G550 \\
\hline Contrast & $W 365$ & G365 & W550 & $G 550$ \\
\hline \multicolumn{5}{|l|}{ C1. Cruzados ${ }^{1}$ vs. Nelore } \\
\hline C1. Crossbreed vs Nellore & $24 * * *$ & $71 * * *$ & $36 * * *$ & 13 \\
\hline C2. 1CN1NL vs. (1BA1NL+1BV1NL) & $8 * * *$ & $11 * * *$ & $16 * * *$ & $30 * *$ \\
\hline C3.1BV1NL vs.1BA1NL & 3 & -20 & $8 * *$ & $33 * *$ \\
\hline C4. 1SM1NL vs. 1CN1NL & $10 * * *$ & $33 * *$ & $7 * *$ & -3 \\
\hline \multicolumn{5}{|c|}{$\begin{array}{l}1 \text { 1/2 Brangus (pelagem preta) }+1 / 2 \text { Nelore (1BA1NL), 1/2 Brangus (pelagem vermelha) }+1 / 2 \text { Nelore } \\
\text { (1BV1NL), 1/2 Canchim + 1/2 Nelore (1CN1NL), 1/2 Simental }+1 / 2 \text { Nelore (1SM1NL). }\end{array}$} \\
\hline \multicolumn{5}{|c|}{$\begin{array}{l}1 / 2 \text { Black Brangus + 1/2 Nellore (1BA1NL), 1/2 Red Brangus + 1/2 Nellore (1BV1NL), 1/2 Canchim + 1/2 Nellore (1CN1NL), , } \\
\text { 1/2 Simmental + 1/2 Nellore (1SM1NL) }\end{array}$} \\
\hline
\end{tabular}

maior para os produtos de touros Simental de monta natural que para os produtos oriundos de inseminação artificial dessa mesma raça. O contraste que compara os cruzados da raça sintética Canchim contra os cruzados Aberdeen Angus (Contraste C4, Tabela 5) não foi significativo, possivelmente, devido ao fato de o número de observações para esses grupos ser muito desigual. O número de observações para este arquivo foi: $1 \mathrm{AG} 1 \mathrm{NL}, 321 ; 1 \mathrm{CN} 1 \mathrm{NL}, 54 ; 1 \mathrm{GL} 1 \mathrm{NL}$, 311; NELORE, 44; e 1SM1NL, 120.

Para o arquivo contendo apenas progênies de touros usados em $\mathrm{MN}$, as análises de variância das características e os contrastes entre as médias apresentaram resultados semelhantes à análise conjunta das progênies de touros de IA e MN (Tabelas 2 e 6). Os cruzados Simental foram mais pesados e ganharam mais peso que os cruzados Canchim a um ano de idade (Contraste $\mathrm{C} 4$, Tabela 6). A maior heterozigose proveniente do cruzamento entre vacas Nelore e touros Simental, provavelmente, resulta em animais mais pesados e com maior ganho de peso até um ano de idade. O número de observações para o arquivo de P365 e G365 foi: 1BA1NL, 496; 1BV1NL, 184; 1CN1NL, 476; NELORE, 534; e 1SM1NL, 273. A raça Simental foi mais pesada que a Canchim $(\mathrm{P}<0,01)$ para $\mathrm{P} 550$, entretanto, não houve diferença para G550. O número de observações desse arquivo foi: 1BA1NL，342; 1BV1NL，141；1CN1NL，342; 
NELORE, 288; e 1SM1NL, 198.

Os resultados não foram sensivelmente diferentes dos obtidos para as progênies de touros de IA e MN juntas, quando se consideraram os arquivos separados de acordo com o tipo de acasalamento (IA e MN). As análises de variância mostraram que não houve vantagem ao se separarem os arquivos, já que os $\mathrm{R}^{2}$ não foram, pronunciadamente, diferentes dos obtidos na análise conjunta para as características estudadas (Tabela 2). Para as análises contendo apenas as progênies de touros de IA, os $\mathrm{R}^{2}$ para $\mathrm{P} 365$, G365, P550 e G550 foram 52, 65, 46 e 51\%, respectivamente. Para as análises contendo apenas as progênies de touros de $\mathrm{MN}$, os $\mathrm{R}^{2}$ para $\mathrm{P} 365, \mathrm{G} 365$, P550 e G550 foram 48, 64, 51 e 49\%, respectivamente. Assim, a análise conjunta dos arquivos de IA e MN possibilita a comparação entre todas as raças utilizadas nos acasalamentos, com a vantagem de apresentar maior número de observações por classe de grupo genético.

Os resultados obtidos neste estudo mostraram a superioridade, no desempenho, dos animais cruzados sobre os puros da raça Nelore para características de crescimento após a desmama, concordando com os resultados obtidos por outros autores no Brasil.

\section{Conclusões}

Os animais cruzados foram mais pesados e ganharam mais peso que os puros da raça Nelore.

Os animais cruzados $\mathrm{F} 1$, de raças continentais, foram mais pesados aos 365 dias e ganharam mais peso até os 365 dias de idade que os demais grupos, entretanto, não diferiram aos 550 dias dos cruzados F1 Aberdeen Angus, sugerindo existir, nas condições do presente estudo, limitações na manutenção de animais cruzados de raças continentais até idades mais avançadas.

A utilização de touros em monta natural das raças sintéticas Brangus e Canchim, em cruzamento com vacas Nelore, foi uma opção viável para produzir animais mais pesados e com maior ganho médio diário entre 365 e 550 dias de idade que os puros da raça Nelore.

\section{Referências Bibliográficas}

CAMPOS, L.T., FRIES, L.A Efeitos ambientais sobre o ganho pós-desmama em bovinos de corte. I) Idade da mãe. In: REUNIÃO ANUAL DA SOCIEDADE BRASILEIRA DE ZOOTECNIA, 26, 1989, Porto Alegre. Anais...Porto Alegre: SBZ, 1989. p. 367.
CUBAS, A.C., PEROTTO, D., ABRAHÃO, J.J.S. et al. Desempenho ponderal de animais Nelore e cruzas com Nelore. I. período pós-desmama. In: REUNIÃO ANUAL DA SOCIEDADE BRASILEIRA DE ZOOTECNIA, 34, 1996, Fortaleza. Anais... Fortaleza: SBZ, 1996. p.127

FRANKE, D.E., 1980. Breed and heterosis effects of american Zebu catlle. J. Anim. Sci., 50 (6):1206-1214.

FRIES, L.A. Cruzamentos em gado de corte. In: SIMPÓSIO SOBRE PECUÁRIA DE CORTE, 4, 1996, Piracicaba. Anais...Piracicaba: FEALQ, 1996, p.109-128.

FRISCH, J.E., 1987. Phisiological reasons for heterosis in growth of Bos indicus x Bos taurus. J. Agric. Sci., 109:213-230.

GREGORY, K.E, TRAIL, J.C.M., MARFLES, H.J.S. et al., 1985. Characterization of breeds of Bos indicus and Bos taurus cattle for maternal and individual traits. J. Anim. Sci., 60 (5):1165-1174.

JOSÉ, W.P.K., CUBAS, A.C., MELLA, S.C. et al. Desenvolvimento ponderal de bovinos Nelore e mestiços (fêmeas), no noroeste do Paraná. In: REUNIÃO ANUAL DA SOCIEDADE BRASILEIRA DE ZOOTECNIA, 28, 1991, João Pessoa. Anais... João Pessoa: SBZ, 1991. p.537.

JOSÉ, W.P.K., MELLA, S.C., CUBAS, A.C. et al. Desenvolvimento ponderal de bovinos Nelore e mestiços (machos), no noroeste do Paraná. In: REUNIÃO ANUAL DA SOCIEDADE BRASILEIRA DE ZOOTECNIA, 28, 1991, João Pessoa. Anais... João Pessoa: SBZ, 1991. p.537.

NITTER, G., 1978. Breed utilization for meat production in sheep. Animal Breeding Abstracts, 46(5):131-143.

PASCHAL, J.C., SANDERS, J.O., KERR, J.L. et al. 1994. Postweaning and feedlot growth and carcass characteristics of Angus, Gir, Indu-Brasil, Nellore and Red Brahman sired F1 calves. J. Anim. Sci., 73:373-380.

RIBEIRO, J.A.R., MACHADO L.C.P., TROVO, J.B.F. et al. Avaliação do desenvolvimento ponderal de novilhas Crioulo Lageano (CL), Nelore (NE) e Charolês (CH) em campos naturais do planalto catarinense. In: REUNIÃO ANUAL DA SOCIEDADE BRASILEIRA DE ZOOTECNIA, 27, 1990, Campinas. Anais ...Campinas: SBZ, 1990. p.473.

SAS Institute Inc., SAS/STAT. 1992. User's Guide, version 6, 4 ed, v.2, Cary: SAS Institute Inc. 842p.

SHERIDAN, A.K. 1981. Crossbreeding and heterosis. Anim. Breeding Abst., 49:131-144

SYRSTAD, O. 1985. Heterosis in Bos Taurus x Bos Indicus Crosses. Liv. Prod. Sci., 12:299-307. 